\title{
Some properties of electrochemical nanostructures ${ }^{\dagger}$
}

\author{
E SANTOS $^{1,2}$, P QUAINO $^{2}$, GERMAN SOLDANO ${ }^{2}$ and W SCHMICKLER ${ }^{2}$ \\ ${ }^{1}$ Faculdad de Matem'atica, Astronom'ia y F'isica, IFFaMAF-CONICET, \\ Universidad Nacional de Cordoba, Cordoba, Argentina \\ ${ }^{2}$ Institute of Theoretical Chemistry, Ulm University, D-89069 Ulm, Germany \\ e-mail: wolfgang.schmickler@uni-ulm.de
}

\begin{abstract}
The physical and electronic properties of several platinum nanostructures have been investigated by density functional calculations. Particular attention has been paid to the structure of the $d$-band. Our results predict, that nanowires and small platinum clusters supported on $\mathrm{Au}(111)$ should be excellent catalysts for the hydrogen evolution reaction; a monolayer of platinum on $\mathrm{Au}(111)$ should also be better than pure platinum.
\end{abstract}

Keywords. Nanowires; work function; cluster models; density of states; hydrogen evolution reaction; electrocatalyst.

\section{Introduction}

In the perpetual search for better catalysts, electrochemists have turned to various metal nanostructures in recent years. Indeed, electrochemistry offers convenient means to generate various nanostructures such as metal overlayers, steps decorated by adatoms, or even monoatomic nanowires. The big scientific challenge is to understand, how the structure a affects the chemical and physical properties of the materials, and how this in turn influences their reactivity. ${ }^{1}$ In this preliminary note, we want to make a contribution to this problem and examine a few structures involving platinum as catalysts, on which much attention has been focused recently. At a first glance, this focus on platinum may appear strange, since this already is a good catalyst. However, there are two good reasons for examining platinum. Firstly, nanostructures usually involve much less of this precious metal than bulk electrodes, so that nanostructures can be much cheaper. Secondly, although reactions such as hydrogen oxidation occur rapidly on platinum, there still seems to be room for substantial improvement. Thus, the standard exchange current density of a fast outer-sphere electron transfer reaction like that of the $\left[\mathrm{Ru}\left(\mathrm{NH}_{3}\right)_{6}\right]^{3+/ 4+}$ is of the order of $100 \mathrm{~A} \mathrm{~cm}^{-2}$, while for the hydrogen evolution on platinum it is only about $10^{-3} \mathrm{~A} \mathrm{~cm}^{-2}$. Since all suitable pure metals and many alloys have

${ }^{\dagger}$ Dedicated to the memory of the late Professor S K Rangarajan

*For correspondence been tested as catalysts, the only hope for improvements lies with nanostructured surfaces. Indeed, experiments with monolayers and islands indicate that these can be more active than bulk materials. ${ }^{5}$

\section{Physical and electronic characteristics}

The systems we want to compare are a pure $\mathrm{Pt}(111)$ surface, a monolayer of platinum on $\mathrm{Au}(111)$, a straight, monoatomic Pt nanowire, and small clusters of $\mathrm{Pt}$ on $\mathrm{Au}(111)$. Although we have not yet concluded our study and determined the catalytic properties of these structures, we have obtained some interesting results, which we would greatly have liked to share and discuss with the late Prof. Rangarajan. All calculations reported have been performed with the dacapo ${ }^{6}$ code; computational details are given elsewhere. ${ }^{7}$

The nearest neighbour distances $d_{\text {nn }}$ between the platinum atoms differ in these systems. On the pure $\operatorname{Pt}(111)$ surface, we have $d_{\mathrm{nn}}=2.831 \AA$, for Pt/ $\mathrm{Au}(111)$ it is determined by the lattice constant of gold, and therefore slightly stretched: $d_{\mathrm{nn}}=2 \cdot 885^{\circ} \AA$. In contrast, it is much shortened for the nanowire: $d_{\mathrm{nn}}=2.391 \AA$. In the wire, each atom has only two nearest neighbours, therefore the bonds between atoms are stronger, and the bond distance has shrunk. The lower coordination in the wire entails a lower binding energy: $E_{b}=-3.034 \mathrm{eV}$ for the wire, compared with $E=-5.709 \mathrm{eV}$ for the bulk (all values are given as calculated by us). 
In a previous publication ${ }^{8}$ it had been observed, that the work functions of monoatomic $\mathrm{Ag}$ and $\mathrm{Au}$ wires are much larger than those of the bulk metal. We find the same effect for platinum. Table 1 gives the work functions for the $f c c(111)$ surfaces and the nanowires of several common electrode materials. In all case, the work function for the nanowire is substantially higher. Since the work function is proportional to the potential of zero charge (pzc), this entails that the pzc of the nanowires is shifted to substantially higher values; of the metals investigated, this effect is largest for gold, where the shift is more than $1 \mathrm{eV}$. Consequently, monoatomic nanowires in electrochemical aqueous solutions carry a negative excess charge over the larger part of the accessible potential range.

For the catalytic properties of metals, the position and width of the $d$ band and its interaction with the reactant are of paramount importance..$^{9-11}$ Naturally, these depend also on the coordination of the atoms and the interatomic spacing. In figure 1 we compare the $d$ band densities of state (DOS) for bulk platinum, for the (111) surface, and the nanowire. The distribution of the DOS is most even for the bulk, where it extends from about $-7 \mathrm{eV}$ to $1 \mathrm{eV}$ with re-

Table 1. Work function for nanowires and $f c c(111)$ surfaces of a few metals.

\begin{tabular}{lcccc}
\hline F/eV & $\mathrm{Ag}$ & $\mathrm{Au}$ & $\mathrm{Cu}$ & $\mathrm{Pt}$ \\
\hline Wire & $5 \cdot 124$ & $6 \cdot 43$ & $5 \cdot 24$ & $6 \cdot 167$ \\
(111) surface & 4.494 & $5 \cdot 261$ & 4.50 & $5 \cdot 701$ \\
\hline
\end{tabular}



Figure 1. $d$ band density of states for $\operatorname{Pt}(111)$, for a monolayer $\mathrm{Pt} / \mathrm{Au}(111)$, and for the cluster of $\mathrm{Pt}$ on $\mathrm{Au}(111)$ shown in figure 2 . spect to the Fermi level. On $\operatorname{Pt}(111)$ the DOS is similar, but shifted to somewhat higher values. In particular, the DOS is low at the lower energy range. In both case, the $d$ band is almost, but not fully, occupied. For the nanowire, the DOS is quite different. There are two major peaks near $-0.5 \mathrm{eV}$ and $-1.5 \mathrm{eV}$, and a smaller one near $-4 \mathrm{eV}$; in addition, the band extends with a low density down to $-7 \mathrm{eV}$. Further examination shows that the two higher peaks are formed by the $d$ and $d_{x y}$ and $d_{x^{2} y^{2}}$ states, where the $z$ axis has been chosen along the wire. The peak near $-4 \mathrm{eV}$ is composed of the $d_{x z}$ and $d_{y z}$ states, while the lowest part consists of the $d_{z^{2}}$ orbitals, whose DOS extends over the full range of the DOS. Thus, the $d_{x y}$ and $d_{x^{2} y^{2}}$ states retain a localized nature, while the $d$ states that have components along the $z$ axis are delocalized. These findings are in accordance with chemical intuition. We note that the occupation of the $d$ band is a little smaller for the nanowire than for the bulk metal, indicating a small change in the mixing between $d$ and $s p$ states.

Next, we want to compare the $\operatorname{Pt}(111)$ surface with two other nanostructures: a monolayer of $\mathrm{Pt}$ on $\mathrm{Au}(111)$, and a small, three atom cluster of $\mathrm{Pt}$ on $\mathrm{Au}(111)$ shown in figure 2. The DOS of these structures are shown in figure 3 . The largest effect occurs for the clusters. Here the DOS is strongly shifted towards the Fermi level, with a pronounced peak lying just below $F$. On the monolayer, the DOS is also strongly shifted towards the Fermi level, but the effect is not quite as large as for the clusters. The shift of the DOS for the monolayer is well explained by the $\mathrm{d}$ band model of Hammer and Nørskov: ${ }^{12}$ Since the lattice constant for the monolayer is smaller than the bulk, the width of the $d$ band is reduced. Since the total occupation of this band does not change, its center has to move towards the Fermi level.

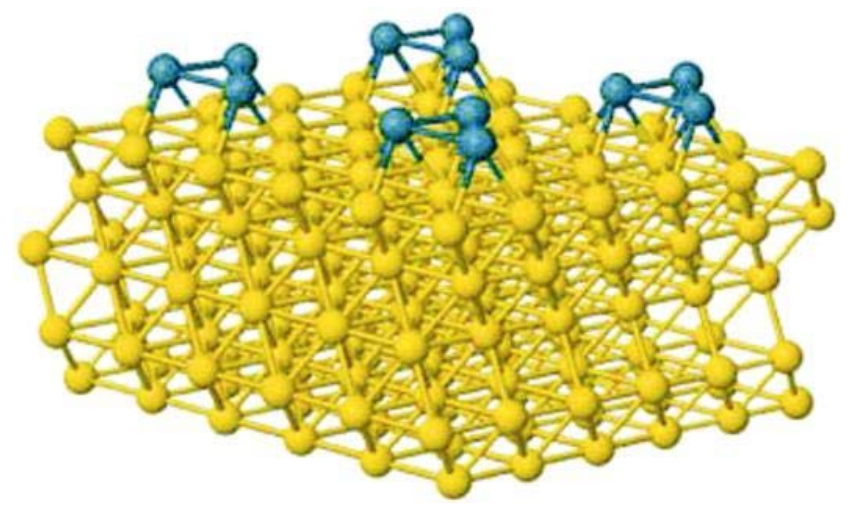

Figure 2. Three atom cluster of Pt on $\mathrm{Au}(111)$. 


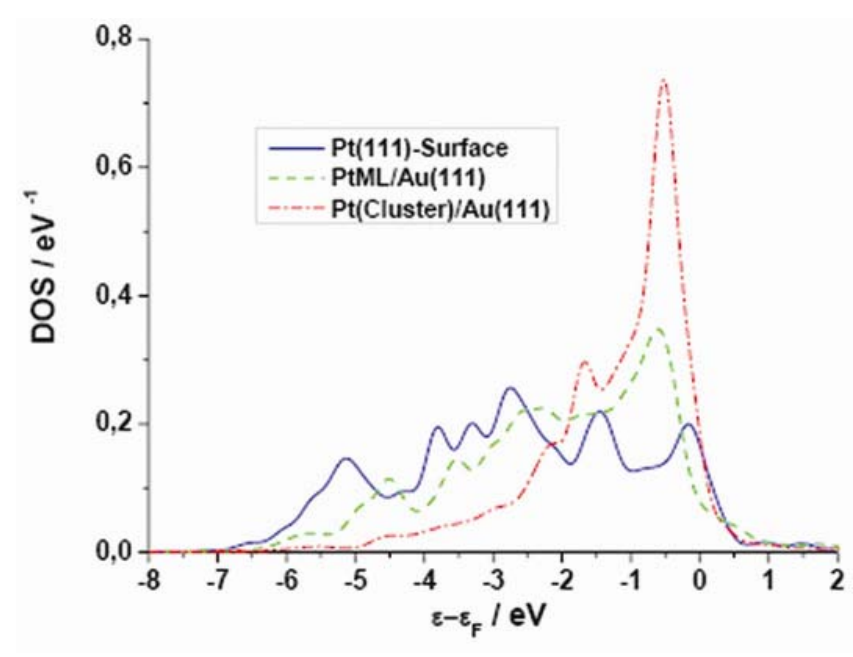

Figure 3. $d$ band density of states for bulk platinum, for $\operatorname{Pt}(111)$, and for a Pt nanowire.

On the basis of these early results, can we predict, which of these structures are the best catalysts for hydrogen evolution or oxidation? On the basis of the position and width of the $\mathrm{d}$ bands alone, we would predict that the nanowires and the clusters are the best catalysts, and the monolayer $\mathrm{Pt} / \mathrm{Au}(111)$ should also be better than pure platinum. Of course, the coupling of the $d$ bands is also important, but according to our preliminary results this does not vary much between the systems investigated. Experimental data for hydrogen evolution show indeed, that $\mathrm{Pt} / \mathrm{Au}(111)$ is a better catalyst that pure $\mathrm{Pt}$, and that for sub-monolayer coverages the rate per atom increases. ${ }^{13}$ Thus, these experimental findings seem to support our predictions, but to obtain definite conclusions we have to study the mechanism of catalysis in greater details.

\section{Conclusion}

Te influence of d-bands in dictating physical and electronic properties of platinum nanoclusters is pointed out. Platinum nanowires and small platinum clusters on $\mathrm{Au}(\mathrm{III})$ are predicted to be efficient electrocatalysts for hydrogen evolution reaction.

\section{Acknowledgements}

The financial support by the Deutsche Forschungsgemeinschaft (Schm 344/34-1 and Sa 1770/1-1), and of the European Union under COST is gratefully acknowledged. E S thanks CONICET for continued support.

\section{References}

1. A good review of the relation between nanostructure and reactivity is given in: A. Groß, Adsorption at nanostructured surfaces, Chapter 89 of Handbook of Theoretical and Computational Nanotechnology (eds) Michael Rieth and Wolfram Schommers (American Scientific Publishers) 2006

2. Iwasita T, Schmickler W and Schultze J W 1985 Ber. Bunsenges. Phys. Chem. 89138

3. Santos E, Iwasita T and Vielstich W 1986 Electrochim. Acta $\mathbf{3 1} 431$

4. Trasatti S 1977 Adv. Electrochem. Electrochem. Eng. 10213

5. see e.g. Pandelov S and Stimming U 2007 Electrochim. Acta 525548 and references therein

6. Hammer B, Hansen L B and. Nørskov K 1999 Phys. Rev. B59 7413; http://www.fysik.dtu.dk/campos

7. Santos E, Lundin A, Pötting K, Quaino $P$ and Schmickler W J. Sol. State Electrochem.; DOI 10.1007/s10008-008-0702-4

8. Leiva E, Vélez P, Sanchez C and Schmickler W 2006 Phys. Rev. B74 035422

9. Hammer B and Nørskov J K 1995 Surf. Sci. 343 211

10. Santos E and Schmickler W 2006 Chem. Phys. Chem. 72282

11. Santos E and Schmickler W 2007 Chem. Phys. 332 39

12. Hammer B and Nørskov J K 2000 Adv. Cat. 4571

13. Quaino P, Santos E and Stimming U (in preparation) 